\title{
A Circular Reflectarray for OAM Generation at Terahertz Regime for 6G Applications
}

\author{
Ali Jihad Ali ${ }^{1}$, Mohsen Khalily ${ }^{1}$, Ali Araghi ${ }^{1}$, Seyed Ehsan Hosseininejad ${ }^{1,2}$, Rahim Tafazolli ${ }^{1}$ \\ $5 G$ \& 6 G Innovation centers (5GIC \& 6GIC), Institute for Communication Systems (ICS) \\ University of Surrey, Guildford, UK \\ Department of Electrical and Computer Engineering, Babol Noshirvani University of Technology, Babol, Iran \\ \{ali.ali,m.khalily\}@surrey.ac.uk
}

\begin{abstract}
A circular reflectarray antenna (RA) for generating Orbital Angular Momentum (OAM) modes in the Terahertz (THz) band is introduced. An interlaced unit cell is proposed to reach a phase variation of $328^{\circ}$ at $185 \mathrm{GHz}$ to $188 \mathrm{GHz}$. Combining RA, OAM, and THz technologies in one structure can be utilized to reach the future requirements of $6 \mathrm{G}$ networks. That is due to the additional degree of freedom that OAM beams can provide for data multiplexing in short-distance wireless communication.
\end{abstract}

\section{INTRODUCTION}

The rapid increase in data rates resulted from the growing demand for mobile and broadband services has urged the extension towards new spectral frequencies in the terahertz $(\mathrm{THz})$ band. It has been predicted that the peak data rate of 6G networks will fall between $100 \mathrm{~Gb} / \mathrm{s}$ and $1 \mathrm{~Tb} / \mathrm{s}$ compared to the $1 \mathrm{~Gb} / \mathrm{s}$ of the $5 \mathrm{G}$ networks. Thus, the microwave and millimetre-wave bands are hardly able to meet the requirements of $6 \mathrm{G}$-and-beyond networks. The $\mathrm{THz}$ band provides ultra high-bandwidth and ultra-low latency to satisfy the requirements of $6 \mathrm{G}$ applications for indoor communications such as wireless brain-computer interactions $(\mathrm{BCI})$ and the internet of everything (IoE). Nevertheless, the inherent lowefficiency of antennas in the $\mathrm{THz}$ band raises concerns related to the improper radiations and low-gain characteristics [1]. Since the periodic and quasi-periodic structures [2] offer highgain antennas in the microwave and $\mathrm{THz}$ ranges, they can be adopted to enhance the antenna efficiency in the $\mathrm{THz}$ band [3]. Reflectarray antennas (RAs) as arrays of unit cells combine the advantages of the parabolic reflectors and the phased array antennas in terms of the high efficiency and low profile, respectively.

The limited bandwidth is considered the major challenge associated with RAs. For this reason, different technologies have been considered to increase the throughput such as the frequency reuse [4] and polarization reuse [5], which may cause interference issues in the communication system. Whereas, the inherent orthogonality among the orbital angular momentum (OAM) beams can be utilized to encode data into different OAM modes using the same frequency so that the system capacity increases. Moreover, spatial multiplexing would significantly solve the interference obstacle that other technologies suffer from, so a reduced number of stages to cancel the channels' interference during the de-multiplexing process would be required. Consequently, the OAM could be regarded as the cornerstone to increase the channel capacity and spectral efficiency of 6G-and-beyond networks, which may supply peak data rates of fifty times faster than $5 \mathrm{G}$ networks. For OAM beams generation, numerous antennas were introduced in the literature as the spiral phase plates (SPP), substrate integrated waveguide antennas, and circular array antennas. Here, we present a RA with an interlaced unit cell. For brevity, only two modes $(l=2$ and $l=3)$ of the proposed RA are simulated and discussed in this paper.

\section{Antenna Configuration And Design}

The presented RA is comprised of a conical horn antenna, a transmission medium (free space), and an array of unit cells. It is worth noting that the desired beam will be produced based on the spiral phase distribution on the quasi-periodic structure. Obtaining OAM beams require a phasic transformation of $2 \pi l$, where $l$ represents the topological charge (the mode number).

\section{A. Geometry of Unit Cell}

The proposed interlaced unit cell is shown in Fig. 1, which consists of four patches and dipoles. It is printed on a Rogers

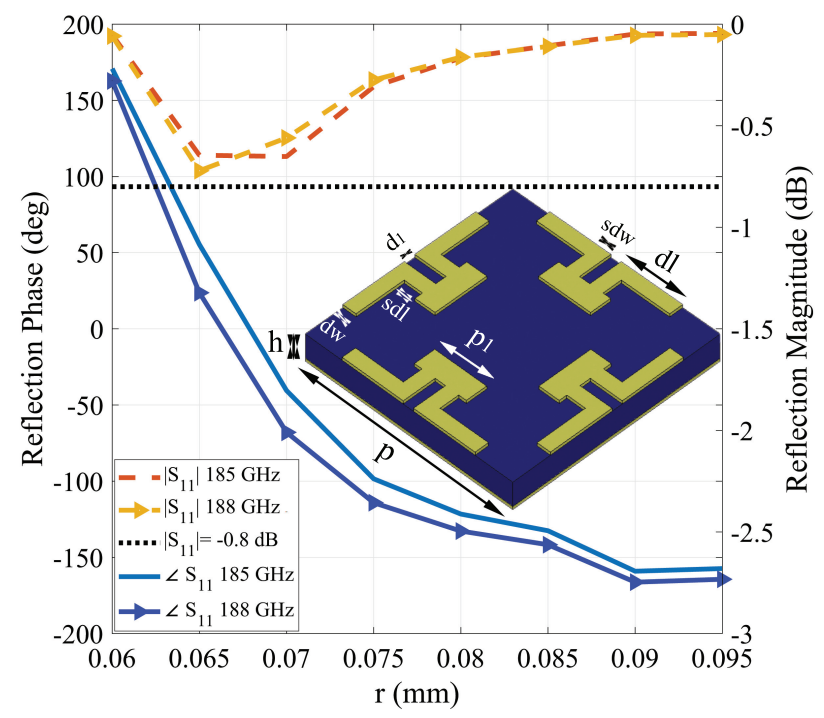

Fig. 1. The schematic and reflection responses of the unit cell. 


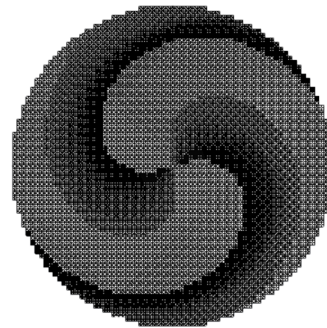

(a)

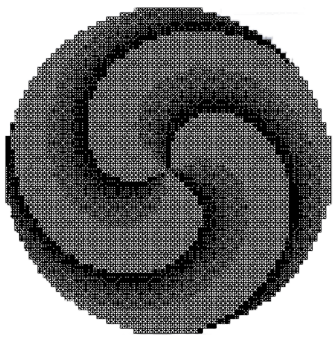

(e)

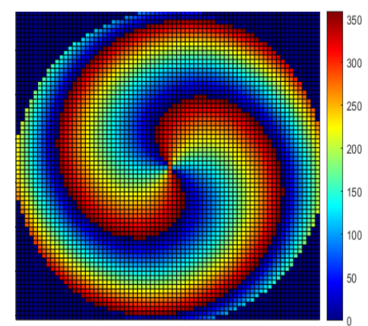

(b)

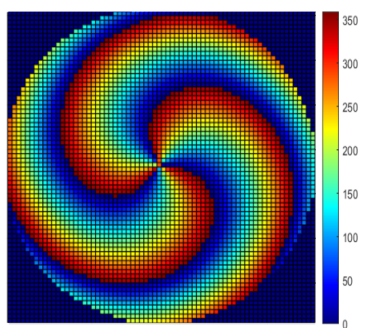

(f)

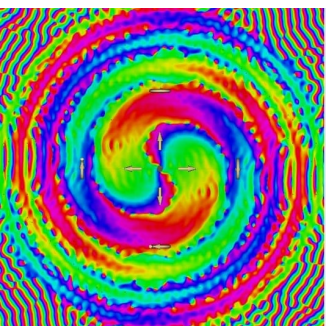

(c)

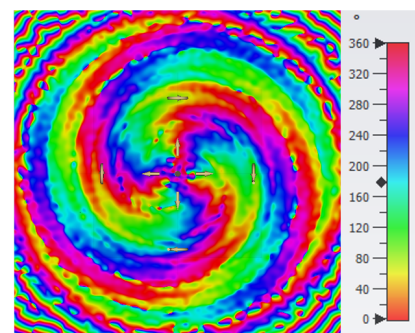

(g)

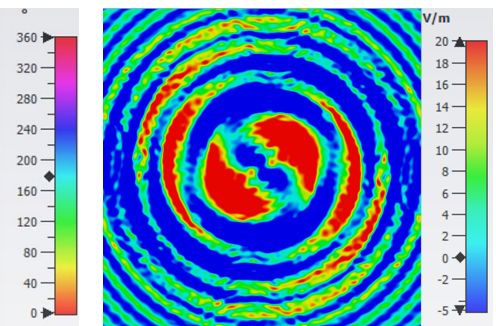

(d)

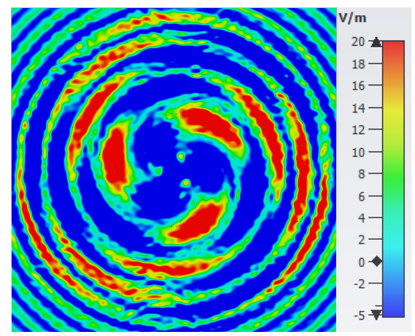

(h)

Fig. 2. Simulated OAM beams with modes $l=+2$ and $l=+3$ : (a) \& (e) layouts of RAs (b) \& (f) spiral phase distributions on the RAs (c) \& (g) E-field phase distributions (d) \& (h) E-field magnitude distributions.

RO3003 dielectric substrate with a thickness of $h=0.08 \mathrm{~mm}$. The dimensions and geometries of the proposed element are as follows: $p=0.68 \mathrm{~mm}, p_{1}=p / 8, t=t_{1}=0.01 \mathrm{~mm}, d_{1}=$ $0.5 \times r, s d w=0.9 \times r, s d l=0.14-0.5 \times r, d w=0.65 \times r$, and $d l=3 \times r$. The parameter $r$ (see Fig. 1) varies between $0.06 \mathrm{~mm}$ and $0.095 \mathrm{~mm}$ to achieve a phase range of around $328^{\circ}$ for frequencies between $185 \mathrm{GHz}$ and $188 \mathrm{GHz}$, which is sufficient phase range to generate the desired beam since it is above $300^{\circ}$ [6]. Fig. 1 also displays the reflection phase and magnitude as functions of $r$ at $185 \mathrm{GHz}$ and $188 \mathrm{GHz}$, respectively. That is obtained by CST Studio Suite software with the periodic boundary condition and Floquet excitation.

\section{B. Simulation Results of $R A$}

With the macroscopic layout of modulated patches, as shown in Fig. 2 (a) and (e), the required phase distribution over the aperture for both modes $l=+2$ and $l=+3$ follows the spiral phase distributions exhibited in Fig. 2 (b) and (f), respectively. The resulted RA has a circular shape with a diameter of $50 \mathrm{~mm}$, which is obtained by a MATLAB code based on the reflection phase response. The desired phase for $m n^{t h}$ element of a RA with mode equals $l$ is given by [7]:

$$
\psi_{m n}=k\left(R_{m n}-\vec{r}_{m n} . \hat{u}_{b}\right)+l \times \arctan \left(y_{n} / x_{m}\right)+\psi_{0}
$$

where $k$ is the free-space wavenumber, $R_{m n}$ is the distance between the desired element and the feeder. Whereas $\hat{u}_{b}$ represents the beam direction, the point $\left(x_{m}, y_{n}\right)$ is the element's position, $\vec{r}_{m n}$ stands for its vector, and $\psi_{0}$ is a constant phase.

The spiral phase distributions of the electric field for $l=+2$ and $l=+3$ in a transverse plane are shown in Fig. 2 (c) and (g). Moreover, Fig. 2 (d) and (h) depict the simulated magnitude of the proposed RA at $188 \mathrm{GHz}$ where an intensity null can be observed at the center of the monitoring plane.
Therefore, the results confirm that OAM beams are generated by the proposed reflectarray antennas since the two conditions of OAM generation are verified, which are the spiral phase front and doughnut intensity profile.

\section{CONCLUSION}

A reflectarray antenna with an interlaced unit cell is proposed to generate OAM beams. The simulation results were presented for two modes $(l=+2 \& l=+3)$ at $188 \mathrm{GHz}$. The proposed antenna can be considered as a suitable candidate to be deployed for ultra-high-speed $\mathrm{THz}$ indoor communications.

\section{REFERENCES}

[1] Sharifi, R., Basiri, R. \& Zareian-Jahromi, E. Optimization-based design of a single-layer wideband reflectarray antenna in the terahertz regime. J Comput Electron 19, 469-481 (2020).

[2] A. Araghi, M. Khalily, P. Xiao, R. Tafazolli, Holographic-based mmwwideband bidirectional frequency scanning leaky wave antenna, 14th European Conference on Antennas and Propagation (EuCAP), 2020.

[3] Hosseininejad, Seyed Ehsan, et al. "Digital metasurface based on graphene: An application to beam steering in terahertz plasmonic antennas." IEEE Transactions on Nanotechnology 18 (2019): 734-746.

[4] D. Martinez-de-Rioja, E. Martinez-de-Rioja and J. A. Encinar, "Multibeam reflectarray for transmit satellite antennas in Ka band using beam-squint," 2016 IEEE International Symposium on Antennas and Propagation (APSURSI), Fajardo, 2016, pp. 1421-1422.

[5] Zhang, X. G., Yu, Q., Jiang, W. X., Sun, Y. L., Bai, L., Wang, Q., Qiu, C.-W., Cui, T. J., Polarization-Controlled Dual-Programmable Metasurfaces. Adv. Sci. 2020, 7, 1903382.

[6] Nayeri, P., Yang, F., \& Elsherbeni, A. (2018). Reflectarray Antennas: Theory, Designs, and Applications Reflectarray Antennas: Theory, Designs, and Applications.

[7] B. Mohammadi, J. Nourinia, C. Ghobadi, F. Alizadeh and M. Karamirad, "Wideband Sub-Wavelength Orbital Angular Momentum Reflectarray Antenna," 2019 5th Conference on Knowledge Based Engineering and Innovation (KBEI), Tehran, Iran, 2019, pp. 869-873. 